\title{
Performance Analysis of Conventional Detection in BFWA Systems
}

\author{
Pei Xiao, Rolando Carrasco \\ School of Electrical, Electronic and Computer Engineering \\ University of Newcastle Upon Tyne NE1 7RU, United Kingdom \\ E-mail: pei.xiao, r.carrasco@ncl.ac.uk \\ Ian Wassell \\ Lab for Communication Engineering, University of Cambridge \\ 15 JJ Thomson Avenue, CB3 0FD, United Kingdom \\ E-mail: ijw24@eng.cam.ac.uk
}

\begin{abstract}
The systems under study are broadband wireless fixed access (BFWA) systems over multipath fading channels. Conventional detection methods like coherent and non-coherent detection are examined theoretically for both QPSK and 16-QAM modulated BFWA systems in this paper and shown to yield unsatisfactory performance. The theoretical analysis for different algorithms are validated by Monte-Carlo simulations and proved to be accurate. They give us an insight into the physical limitations of the BFWA channels and suggest solutions to improve the capacity and performance of future BFWA systems.
\end{abstract}

\section{INTRODUCTION}

It is becoming apparent that access to the Internet is of growing economic and political importance. It is also clear that low bandwidth dial-up Internet access is restricting the services and applications that can be offered. What is required is a quantum leap in access bandwidth to free up the Internet for innovative applications. One possible solution is to use the existing local-loop. This approach requires the installation of digital subscriber line (DSL) equipment at the exchange and customer premises. Unfortunately, the length and quality of the local-loop infrastructure will prevent this service being offered universally. Another option for providing broadband access is via cable TV networks. However, the availability of these services is far from universal and contention will seriously degrade throughput per user. An alternative approach is to deploy broadband fixed wireless access (BFWA) technology. The advantage of such an approach is that it enables operators in a competitive environment to roll-out broadband services in a rapid and cost efficient manner. BFWA networks generally employ a pointto-multi-point architecture [1], where a single based station (BS) communicates with many subscriber units (SU) placed at the user locations. Standardization of BFWA systems is currently undertaken by the IEEE 802.16 working group [2] and the ETSI HIPERMAN group [3].

One of the limiting factors in outdoor wireless transmission is the multipath channel between the transmitter and the receiver causing itersymbol interference (ISI), which degrades the system performance and limits the maximum achievable data rate. In this paper, we provide a theoretical approach to analyze the effect of ISI on the performance of the BFWA systems, with an attempt to gain a deep insight into physical limitations of the BFWA channels with conventional detection techniques. The multipath channel can be modeled by an equivalent baseband system where the transmit filter, the channel and the receive filter, are represented by a discrete-time $L$-tap transversal filter with finite-length impulse response $h_{n}=\sum_{l=0}^{L-1} h_{l} \delta_{n-l}$ where $h_{l}$ denotes the complex channel coefficients. Tailored for different terrain conditions, a set of 6 typical channel models called Stanford University Interim (SUI) Channel Models used for simulation, design, development and testing of technologies suitable for fixed broadband wireless applications were proposed in [4]. All of them are simulated using 3 taps, having either Rayleigh or Ricean amplitude distributions. For the purpose of this study, we select SUI-3 channel with tap spacing of 500ns, and maximum tap delay at 1000ns. To simplify the analysis, we assume the transmitted data rate is $4 \mathrm{Mbps}$ with QPSK modulation or $8 \mathrm{Mbps}$ WITH 16-QAM modulation so that the multipath fading is modelled as a tapped-delay line with adjacent taps equally spaced at symbol rate. The received signal is formed as

$$
r_{n}=h_{0} s_{n}+h_{1} s_{n-1}+h_{2} s_{n-2}+v_{n}
$$

where the channel coefficients $h_{0}, h_{1}, h_{2}$ are complex Gaussian distributed and assumed to remain constant during the transmission of one block of data. They, however, vary from block to block. The amplitude of the first tap $\left|h_{0}\right|$ is characterized by a Ricean distribution due to the line of sight propagation. The amplitudes of the taps $\left|h_{1}\right|,\left|h_{2}\right|$ are Rayleigh distributed. The transmitted PSK/QAM symbol at time instant $n$ is denoted as $s_{n}=x_{n}+j y_{n}$, and $v_{n}$ is the complex additive white Gaussian noise with zero mean and variance $N_{0}$.

\section{Performance of Coherent Detection}

Denote $\hat{h}_{0}$ as an estimate of $h_{0}$, and assume it is an accurate estimate of $h_{0}$, i.e., $\hat{h}_{0} \approx h_{0}$. To detect the transmitted symbols coherently, we multiply the received signal with the conjugate of $\hat{h}_{0}$, i.e.,

$$
\begin{aligned}
r_{n}^{\prime} & =\hat{h}_{0}^{*}\left(h_{0} s_{n}+h_{1} s_{n-1}+h_{2} s_{n-2}+v_{n}\right) \\
& =\hat{h}_{0}^{*} h_{0} s_{n}+\underbrace{\hat{h}_{0}^{*}\left(h_{1} s_{n-1}+h_{2} s_{n-2}+v_{n}\right)}_{\text {combined ISI and noise }} \\
& =\hat{h}_{0}^{*} h_{0} s_{n}+w_{n} \approx\left|h_{0}\right|^{2} s_{n}+w_{n}
\end{aligned}
$$




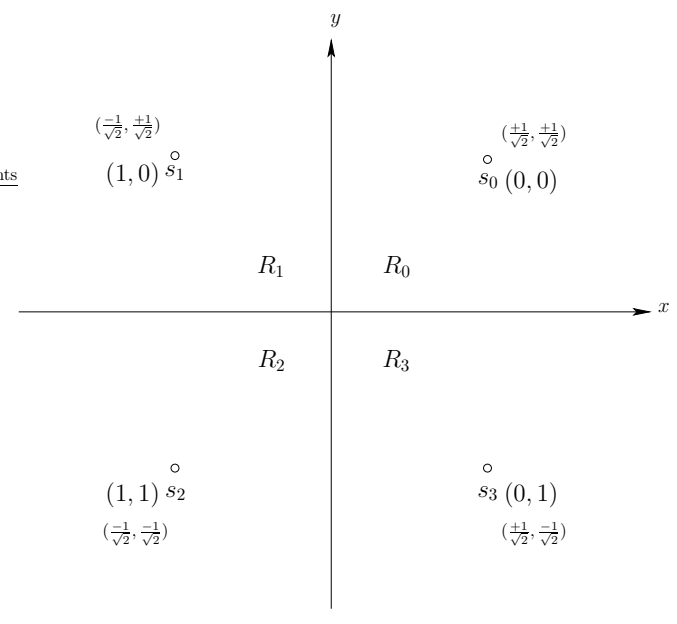

Fig. 1. QPSK constellation and decision regions.

where the combined ISI and noise $w_{n}=w_{I}+j w_{Q}$ is a complex Gaussian random variable with $\mathrm{PDF} \sim \mathcal{C N}\left(0, N_{w}\right)$ and variance $N_{w}=\left|h_{0}\right|^{2}\left(\mathrm{E}\left[\left|h_{1}\right|^{2}\right]+\mathrm{E}\left[\left|h_{2}\right|^{2}\right]+N_{0}\right)=\left|h_{0}\right|^{2}\left(P_{1}+\right.$ $\left.P_{2}+N_{0}\right)$.

\section{A. Coherence detection for QPSK modulation}

QPSK signal constellation is plotted in Fig. 1. Due to the symmetry of the QPSK constellation, the symbol error probability $P_{e}$ equals the conditional error probabilities, i.e., $P_{e}=P\left(e \mid s_{n}=s_{0}\right)=P\left(e \mid s_{n}=s_{1}\right)=P\left(e \mid s_{n}=\right.$ $\left.s_{2}\right)=P\left(e \mid s_{n}=s_{3}\right)$. With maximum likelihood detection, the optimum decision regions are simply four quadrants $R_{0}, R_{1}, R_{2}, R_{3}$. Suppose $s_{0}$ is transmitted, the probability of making correct decision $P\left(c \mid s_{n}=s_{0}\right)$ is the probability of $r_{n}^{\prime}$ falling in the correct decision region $R_{0}$ (the first quadrant), i.e.,

$$
\begin{aligned}
& P\left(c \mid s_{n}=s_{0}\right)=P_{r}\left\{r_{n}^{\prime}=\left|h_{0}\right|^{2} s_{0}+w_{n} \in R_{0}\right\} \\
& =P_{r}\left\{\frac{\left|h_{0}\right|^{2}}{\sqrt{2}}+w_{I}+j\left(\frac{\left|h_{0}\right|^{2}}{\sqrt{2}}+w_{Q}\right) \in R_{0}\right\} \\
& =P_{r}\left\{\frac{\left|h_{0}\right|^{2}}{\sqrt{2}}+w_{I}>0\right\} \cdot P_{r}\left\{\frac{\left|h_{0}\right|^{2}}{\sqrt{2}}+w_{Q}>0\right\} \\
& =P_{r}\left\{w_{I}>-\frac{\left|h_{0}\right|^{2}}{\sqrt{2}}\right\} \cdot P_{r}\left\{w_{Q}>-\frac{\left|h_{0}\right|^{2}}{\sqrt{2}}\right\}
\end{aligned}
$$

The third equality in (1) holds since the Gaussian random variables $w_{I} \sim \mathcal{N}\left(0, N_{w} / 2\right)$ and $w_{Q} \sim \mathcal{N}\left(0, N_{w} / 2\right)$ are statistically independent. Normalizing $w_{I}, w_{Q}$ to unit vari- ance, yield

$$
\begin{array}{r}
P\left(c \mid s_{0}\right)=P_{r}\left\{\frac{w_{I}}{\sqrt{N_{w} / 2}}>-\frac{\left|h_{0}\right|^{2}}{\sqrt{2} \sqrt{N_{w} / 2}}\right\} \\
\cdot P_{r}\left\{\frac{w_{Q}}{\sqrt{N_{w} / 2}}>-\frac{\left|h_{0}\right|^{2}}{\sqrt{2} \sqrt{N_{w} / 2}}\right\} \\
=\left[1-Q\left(\frac{\left|h_{0}\right|^{2}}{\sqrt{\left|h_{0}\right|^{2}\left(P_{1}+P_{2}+N_{0}\right)}}\right)\right]^{2} \\
=\left[1-Q\left(\frac{\left|h_{0}\right|}{\sqrt{P_{1}+P_{2}+N_{0}}}\right)\right]^{2}
\end{array}
$$

where $Q(x)=\int_{x}^{\infty} \frac{1}{\sqrt{2 \pi}} \exp \left(-t^{2} / 2\right) d t$ is the complementary Gaussian cumulative distribution function. The relationship between $P_{e}$ and $P\left(c \mid s_{0}\right)$ is

$$
\begin{aligned}
& P_{e}=P\left(e \mid s_{0}\right)=1-P\left(c \mid s_{0}\right) \\
& =2 Q\left(\frac{\left|h_{0}\right|}{\sqrt{P_{1}+P_{2}+N_{0}}}\right)-Q^{2}\left(\frac{\left|h_{0}\right|}{\sqrt{P_{1}+P_{2}+N_{0}}}\right)
\end{aligned}
$$

We know that $r=\left|h_{0}\right|$ is Ricean distributed with PDF

$$
p(r)=\frac{r}{\sigma^{2}} \exp \left(-\frac{r^{2}+s^{2}}{2 \sigma^{2}}\right) I_{0}\left(\frac{r s}{\sigma^{2}}\right), \quad r \geq 0
$$

where $I_{0}(x)$ is the $0^{\text {th }}$ order modified Bessel function of the first kind [5, p. 44]. For gray coded QPSK, $P_{b} \approx P_{e} / 2$, therefore

$$
P_{b}(r) \approx P_{e}(r) / 2 \approx Q\left(\frac{r}{\sqrt{P_{1}+P_{2}+N_{0}}}\right)
$$

To obtain the error probability when $r$ is random, we must average $P_{b}(r)$ given in (3) over distribution of $r$, i.e.,

$$
\begin{aligned}
& \bar{P}_{b}=\int_{0}^{\infty} P_{b}(r) p(r) d r \\
& =\int_{0}^{\infty} Q\left(\frac{r}{\sqrt{P_{1}+P_{2}+N_{0}}}\right) \frac{r}{\sigma^{2}} \exp \left(-\frac{r^{2}+s^{2}}{2 \sigma^{2}}\right) I_{0}\left(\frac{r s}{\sigma^{2}}\right) d r \\
& =\frac{1}{2} \exp \left(\frac{-s^{2}}{2 \sigma^{2}}\right) \sum_{n=1}^{\infty} g(n)(1-\beta)^{n} \sum_{k=0}^{n-1} 2^{-k}\left(\begin{array}{c}
n-1+k \\
k
\end{array}\right)(1+\beta)^{k}
\end{aligned}
$$

where

$$
\begin{aligned}
g(n) & =\frac{(\sigma s)^{2 n-2}}{4^{n-1} \sigma^{4 n-4}(n-1) !} \\
\alpha & =\sqrt{P_{1}+P_{2}+N_{0}} / \sigma, \quad \beta=\left(\alpha^{2}+1\right)^{-1 / 2}
\end{aligned}
$$

In (4), the derivation of the closed form expression is omitted to conserve space.

\section{B. Coherent detection for 16-QAM}

The 16-QAM constellation and maximum likelihood decision regions are depicted in Fig. 2. The decision regions can be squares (type $x$ ), squares with one open side (type 


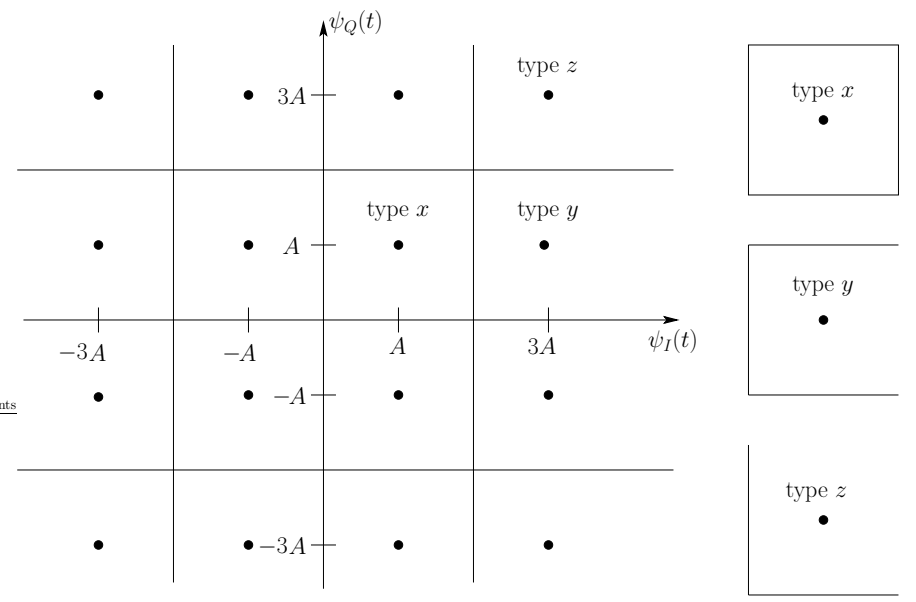

Fig. 2. 16-QAM constellation and decision regions.

$y$ ) or squares with two open sides (type $z$ ), see Fig. 2. To compute the bit error probability, we first need to compute the conditional error probability for these three types of regions. Conditioned on that we send a symbol that has a decision region of type $x, y, z$, and the probability of making a wrong bit decision is denoted by $P_{x}, P_{y}, P_{z}$.

To simplify the calculation, we assume that a symbol error results in maximum two bit errors. If the constellation is gray coded, 2 bit error occurs when the symbol is erroneously decoded to the the symbol in diagonal neighbouring regions; 1 bit error occurs when the symbol is erroneously decoded to the the symbol in non-diagonal neighbouring regions. The probability of these two events are denoted as $P_{x 2}, P_{y 2}, P_{z 2}$, and $P_{x 1}, P_{y 1}, P_{z 1}$ for symbol with decision region of type $x, y, z$. Due to the symmetry of the constellation, $P_{2}=P_{x 2}=P_{y 2}=P_{z 2}$, and $P_{1}=P_{x 1}=P_{y 1}=P_{z 1}$. Given $s_{n}$ is of type $x$ symbol (e.g., the one pointed in Fig. 2), the received signal after phase correction is $r_{n}^{\prime} \approx\left|h_{0}\right|^{2} s_{n}+w_{n}=\left|h_{0}\right|^{2}(A+j A)+w_{n}$. The probabilities $P_{1}, P_{2}$ can be computed as

$$
\begin{aligned}
P_{1} & =P_{r}\left\{w_{I}>A\left|h_{0}\right|^{2}\right\}=P_{r}\left\{w_{I}<-A\left|h_{0}\right|^{2}\right\} \\
& =P_{r}\left\{w_{Q}>A\left|h_{0}\right|^{2}\right\}=P_{r}\left\{w_{Q}<-A\left|h_{0}\right|^{2}\right\} \\
& =Q\left(\frac{A\left|h_{0}\right|^{2}}{\sqrt{N_{w} / 2}}\right) \\
P_{2} & =P_{r}\left\{w_{I}>A\left|h_{0}\right|^{2}, w_{Q}>A\left|h_{0}\right|^{2}\right\} \\
& =P_{r}\left\{w_{I}<-A\left|h_{0}\right|^{2}, w_{Q}<-A\left|h_{0}\right|^{2}\right\} \\
& =P_{r}\left\{w_{I}>A\left|h_{0}\right|^{2}, w_{Q}<-A\left|h_{0}\right|^{2}\right\} \\
& =P_{r}\left\{w_{I}<-A\left|h_{0}\right|^{2}, w_{Q}>A\left|h_{0}\right|^{2}\right\} \\
& =Q^{2}\left(\frac{A\left|h_{0}\right|^{2}}{\sqrt{N_{w} / 2}}\right)
\end{aligned}
$$

The conditional bit error probability $P_{x}$ is computed as

$$
\begin{aligned}
P_{x} & =\frac{1}{k}\left[n_{x 1} \cdot 1 \cdot P_{1}+n_{x 2} \cdot 2 \cdot P_{2}\right]=\frac{1}{4}\left[4 P_{1}+8 P_{2}\right] \\
& =Q\left(\frac{A\left|h_{0}\right|^{2}}{\sqrt{N_{w} / 2}}\right)+2 Q^{2}\left(\frac{A\left|h_{0}\right|^{2}}{\sqrt{N_{w} / 2}}\right)
\end{aligned}
$$

where $k$ stands for the number of bits per symbol (For 16QAM, $k=4$ ), and $n_{x 1}, n_{x 2}$ are the number of neighbouring regions that differ in 1 and 2 bits, respectively, from the transmitted symbol having a decision region of type $x$.

Similarly,

$$
\begin{aligned}
P_{y} & =\frac{1}{k}\left[n_{y 1} \cdot 1 \cdot P_{1}+n_{y 2} \cdot 2 \cdot P_{2}\right]=\frac{1}{4}\left[3 P_{1}+4 P_{2}\right] \\
& =\frac{3}{4} Q\left(\frac{A\left|h_{0}\right|^{2}}{\sqrt{N_{w} / 2}}\right)+Q^{2}\left(\frac{A\left|h_{0}\right|^{2}}{\sqrt{N_{w} / 2}}\right) \\
P_{z} & =\frac{1}{k}\left[n_{z 1} \cdot 1 \cdot P_{1}+n_{z 2} \cdot 2 \cdot P_{2}\right]=\frac{1}{4}\left[2 P_{1}+2 P_{2}\right] \\
& =\frac{1}{2} Q\left(\frac{A\left|h_{0}\right|^{2}}{\sqrt{N_{w} / 2}}\right)+\frac{1}{2} Q^{2}\left(\frac{A\left|h_{0}\right|^{2}}{\sqrt{N_{w} / 2}}\right)
\end{aligned}
$$

where $n_{y 1}, n_{y 2}\left(n_{z 1}, n_{z 2}\right)$ are the number of neighbouring regions that differ in 1 and 2 bits, respectively, from the transmitted symbol having a decision region of type $y(z)$. The bit error probability is thus

$$
\begin{aligned}
P_{b} & =\frac{1}{16}\left(n_{x} P_{x}+n_{y} P_{y}+n_{z} P_{z}\right)=\frac{1}{16}\left(4 P_{x}+8 P_{y}+4 P_{z}\right) \\
& =\frac{3}{4} Q\left(\frac{A\left|h_{0}\right|^{2}}{\sqrt{N_{w} / 2}}\right)+\frac{9}{8} Q^{2}\left(\frac{A\left|h_{0}\right|^{2}}{\sqrt{N_{w} / 2}}\right) \\
& =\frac{3}{4} Q\left(\frac{\sqrt{2} A\left|h_{0}\right|}{\sqrt{P_{1}+P_{2}+N_{0}}}\right)+\frac{9}{8} Q^{2}\left(\frac{\sqrt{2} A\left|h_{0}\right|}{\sqrt{P_{1}+P_{2}+N_{0}}}\right)
\end{aligned}
$$

where $n_{x}, n_{y}, n_{z}$ is the number of $x, y, z$ type regions in the constellation. For 16-QAM, $n_{x}=4, n_{y}=8, n_{z}=4$.

To obtain the error probability when $r=\left|h_{0}\right|$ is random, we must average $P_{b}(r)$ given in (5) over distribution of $r$, i.e.,

$$
\begin{aligned}
\bar{P}_{b}= & \int_{0}^{\infty} P_{b}(r) p(r) d r \\
= & \int_{0}^{\infty}\left[\frac{3}{4} Q\left(\frac{\sqrt{2} A r}{\sqrt{P_{1}+P_{2}+N_{0}}}\right)+\frac{9}{8} Q^{2}\left(\frac{\sqrt{2} A r}{\sqrt{P_{1}+P_{2}+N_{0}}}\right)\right] \\
& \cdot \frac{r}{\sigma^{2}} \exp \left(-\frac{r^{2}+s^{2}}{2 \sigma^{2}}\right) I_{0}\left(\frac{r s}{\sigma^{2}}\right) d r
\end{aligned}
$$

The closed form of (7) is difficult to derive due to the quadratic term of the Q-function. One way of tacking this problem is to use the method proposed by Holtzman in [6]. It gives a simple and accurate method to evaluate the expectation without carrying out the integration. With Holtzman approximation, the solution to our problem becomes

$$
\begin{aligned}
P_{b}(r) & =\frac{3}{4} Q\left(\frac{\sqrt{2} A r}{\sqrt{P_{1}+P_{2}+N_{0}}}\right)+\frac{9}{8} Q^{2}\left(\frac{\sqrt{2} A r}{\sqrt{P_{1}+P_{2}+N_{0}}}\right) \\
\bar{P}_{b} & \approx \frac{2}{3} P_{b}\left(\mu_{r}\right)+\frac{1}{6} P_{b}\left(\mu_{r}+\sqrt{3} \sigma_{r}\right)+\frac{1}{6} P_{b}\left(\mu_{r}-\sqrt{3} \sigma_{r}\right)
\end{aligned}
$$


where $\sigma_{r}=\sqrt{\mathrm{E}\left[r^{2}\right]-\mu_{r}^{2}}=\sqrt{\mathrm{E}\left[\left|h_{0}\right|^{2}\right]-\mu_{r}^{2}}$, and $\mu_{r}$ can be derived as

$$
\begin{aligned}
& \mu_{r}=\frac{\exp \left(-s^{2} / 2 \sigma^{2}\right)}{\sigma^{2}} \int_{0}^{\infty} \exp \left(-\frac{r^{2}}{2 \sigma^{2}}\right) \sum_{k=0}^{\infty} \frac{r^{2 k+2} s^{2 k}}{\left(2 \sigma^{2}\right)^{2 k}(k !)^{2}} d r \quad p(x)=\frac{1}{\sqrt{2 \pi} \sigma} \exp \left[-\frac{x^{2}}{2 \sigma^{2}}\right], \quad-\infty \leq x \leq \infty \\
& =\frac{\exp \left(-s^{2} / 2 \sigma^{2}\right)}{\sigma^{2}} \int_{0}^{\infty} \exp \left(-\frac{r^{2}}{2 \sigma^{2}}\right) \sum_{n=1}^{\infty} \frac{r^{2 n} s^{2 n-2}}{\left(2 \sigma^{2}\right)^{2 n-2}[(n-1) !]^{2}} d r p(y)=\frac{1}{\sqrt{2 \pi} \sigma} \exp \left[-\frac{\left(y-m_{y}\right)^{2}}{2 \sigma^{2}}\right], \quad-\infty \leq y \leq \infty
\end{aligned}
$$

According to $[7$, p. 255]

$$
\begin{aligned}
& \int_{0}^{\infty} x^{2 n} \exp \left(-p x^{2}\right) d x=\frac{(2 n-1) ! !}{2(2 p)^{n}} \sqrt{\frac{\pi}{p}} \\
& p>0, n=0,1,2, \ldots \quad(2 n-1) ! !=1 \cdot 3 \cdot 5 \cdots(2 n-1)
\end{aligned}
$$

Denote $p=\frac{1}{2 \sigma^{2}}$, we have

$$
\begin{aligned}
\mu_{r} & =2 p \exp \left(-p s^{2}\right) \int_{0}^{\infty} \exp \left(-p r^{2}\right) \sum_{n=1}^{\infty} \frac{r^{2 n} s^{2 n-2} p^{2 n-2}}{[(n-1) !]^{2}} d r \\
& =\sqrt{\pi p} \exp \left(-p s^{2}\right) \sum_{n=1}^{\infty} \frac{s^{2 n-2} p^{n-2}(2 n-1) ! !}{2^{n}[(n-1) !]^{2}}
\end{aligned}
$$

\section{Performance of Non-COHEREnt DETECTION}

Non-coherent detection is performed on the original received signal without correcting the phase shift. Let us reform the received signal as

$$
r_{n}=h_{0} s_{n}+\underbrace{h_{1} s_{n-1}+h_{2} s_{n-2}+v_{n}}_{\text {combined ISI and noise }}=h_{0} s_{n}+w_{n}
$$

where $w_{n}=h_{1} s_{n-1}+h_{2} s_{n-2}+v_{n} \sim \mathcal{N}\left(0, N_{w}\right)$ stands for combined ISI and noise, which is Gaussian distributed, and $N_{w}=\mathrm{E}\left[\left|h_{1}\right|^{2}\right]+\mathrm{E}\left[\left|h_{2}\right|^{2}\right]+N_{0}=P_{1}+P_{2}+N_{0}$.

\section{A. Non-coherent detection for $Q P S K$}

The probability of making a symbol error, e.g., $\hat{s}_{n}=s_{1}$ given $s_{0}$ is transmitted can be computed as

$$
\begin{aligned}
& P\left(\hat{s}_{n}=s_{1} \mid s_{n}=s_{0}\right)=P_{r}\left\{h_{0} s_{0}+w_{n} \in R_{1}\right\} \\
& =P_{r}\left\{\left(h_{I}+j h_{Q}\right)\left(\frac{1}{\sqrt{2}}+j \frac{1}{\sqrt{2}}\right)+w_{n} \in R_{1}\right\} \\
& =P_{r}\left\{\frac{h_{I}-h_{Q}}{\sqrt{2}}+w_{I}<0\right\} \cdot P_{r}\left\{\frac{h_{I}+h_{Q}}{\sqrt{2}}+w_{Q}>0\right\} \\
& =Q\left(\frac{h_{I}-h_{Q}}{\sqrt{P_{1}+P_{2}+N_{0}}}\right) Q\left(-\frac{h_{I}+h_{Q}}{\sqrt{P_{1}+P_{2}+N_{0}}}\right)
\end{aligned}
$$

Similarly, other conditional error probabilities can be obtained as

$$
\begin{aligned}
P\left(\hat{s}_{n}\right. & \left.=s_{2} \mid s_{n}=s_{0}\right) \\
& =Q\left(\frac{h_{I}-h_{Q}}{\sqrt{P_{1}+P_{2}+N_{0}}}\right) Q\left(\frac{h_{I}+h_{Q}}{\sqrt{P_{1}+P_{2}+N_{0}}}\right) \\
P\left(\hat{s}_{n}\right. & \left.=s_{3} \mid s_{n}=s_{0}\right) \\
& =Q\left(-\frac{h_{I}-h_{Q}}{\sqrt{P_{1}+P_{2}+N_{0}}}\right) Q\left(\frac{h_{I}+h_{Q}}{\sqrt{P_{1}+P_{2}+N_{0}}}\right)
\end{aligned}
$$

According to the signal constellation plotted in Fig. 1, the error event $\left(\hat{s}_{n}=s_{2} \mid s_{n}=s_{0}\right)$ results in 2 bits error, while both $\left(\hat{s}_{n}=s_{1} \mid s_{n}=s_{0}\right)$ and $\left(\hat{s}_{n}=s_{3} \mid s_{n}=s_{0}\right)$ result in 1 bit error. The relationship between bit error probability and the conditional symbol error probabilities is therefore

$$
\begin{gathered}
P_{b}=\frac{1}{k}\left[2 \times P\left(\hat{s}_{n}=s_{2} \mid s_{n}=s_{0}\right)+1 \times P\left(\hat{s}_{n}=s_{1} \mid s_{n}=s_{0}\right)\right. \\
\left.\quad+1 \times P\left(\hat{s}_{n}=s_{3} \mid s_{n}=s_{0}\right)\right]
\end{gathered}
$$

where $k$ stands for the number of bits per symbol. For QPSK modulation, $k=2$. Based on (9), (10) and (12), the bit error probability is expressed as

$$
\begin{aligned}
& P_{b}(x, y)=\frac{1}{2} Q\left(\frac{x}{\sqrt{N_{w}}}\right) Q\left(\frac{-y}{\sqrt{N_{w}}}\right)+ \\
& \frac{1}{2} Q\left(\frac{-x}{\sqrt{N_{w}}}\right) Q\left(\frac{y}{\sqrt{N_{w}}}\right)+Q\left(\frac{x}{\sqrt{N_{w}}}\right) Q\left(\frac{y}{\sqrt{N_{w}}}\right)
\end{aligned}
$$

where $N_{w}=P_{1}+P_{2}+N_{0}$. The average bit error probability $\bar{P}_{b}$ is derived by taking expectation of $P_{b}(x, y)$ expressed in (13) with respect to $x$ and $y$. Since $x$ is a zero-mean normal random variable, $\mathrm{E}[Q(\lambda x)]=Q(0)=0.5[8, \mathrm{p} .102]$. Therefore, expectation of (13) with respect to $x$ is equivalent to

$$
P_{b}(y)=\frac{1}{4} Q\left(\frac{-y}{\sqrt{N_{w}}}\right)+\frac{3}{4} Q\left(\frac{y}{\sqrt{N_{w}}}\right)
$$

According to [8, p. 102], if $z$ is a zero-mean, unit-variance, normal random variable, then $\mathrm{E}[Q(\mu+\lambda z)]=Q\left(\frac{\mu}{\sqrt{1+\lambda^{2}}}\right)$. Assign $z=\left(y-m_{y}\right) / \sigma$ such that $z$ is a zero-mean, unitvariance, normal random variable. By taking the expectation of (14), we derive the average bit error probability as

$$
\begin{aligned}
\bar{P}_{b} & =\mathrm{E}\left[\frac{1}{4} Q\left(-\frac{\sigma z+m_{y}}{\sqrt{N_{w}}}\right)+\frac{3}{4} Q\left(\frac{\sigma z+m_{y}}{\sqrt{N_{w}}}\right)\right] \\
& =\frac{1}{4} Q\left(\frac{\mu_{1}}{\sqrt{1+\lambda_{1}^{2}}}\right)+\frac{3}{4} Q\left(\frac{\mu_{2}}{\sqrt{1+\lambda_{2}^{2}}}\right)
\end{aligned}
$$

where $\lambda_{1}=-\frac{\sigma}{\sqrt{N_{w}}}, \mu_{1}=-\frac{m_{y}}{\sqrt{N_{w}}}, \lambda_{2}=\frac{\sigma}{\sqrt{N_{w}}}, \mu_{2}=\frac{m_{y}}{\sqrt{N_{w}}}$.

\section{B. Non-coherent detection for 16-QAM}

Given $s_{n}$ is a type $a$ symbol, the received signal

$$
\begin{aligned}
r_{n} & =h_{0} s_{n}+w_{n}=\left(h_{I}+j h_{Q}\right)(A+j A)+w_{n} \\
& =A\left(h_{I}-h_{Q}\right)+w_{I}+j A\left(h_{I}+h_{Q}\right)+j w_{Q}
\end{aligned}
$$

In this case, the probability of 1 error event along I axis 4 is different from the one along $\mathrm{Q}$ axis. They are denoted by 
$P_{1 I}, P_{1 Q}$, respectively. The probability of 2 errors event is denoted as $P_{2}$. These probabilities are computed as

$$
\begin{aligned}
P_{1 I} & =P_{r}\left\{A\left(h_{I}-h_{Q}\right)+w_{I}<0\right\}=P_{r}\left\{w_{I}<-A\left(h_{I}-h_{Q}\right)\right\} \\
& =P_{r}\left\{\frac{w_{I}}{\sqrt{N_{w} / 2}}<-\frac{\sqrt{2} A\left(h_{I}-h_{Q}\right)}{\sqrt{N_{w}}}\right\} \\
& =Q\left[\frac{\sqrt{2} A\left(h_{I}-h_{Q}\right)}{\sqrt{N_{w}}}\right] \\
P_{1 Q} & =P_{r}\left\{A\left(h_{I}+h_{Q}\right)+w_{Q}<0\right\}=P_{r}\left\{w_{Q}<-A\left(h_{I}+h_{Q}\right)\right\} \\
& =P_{r}\left\{\frac{w_{Q}}{\sqrt{N_{w} / 2}}<-\frac{\sqrt{2} A\left(h_{I}+h_{Q}\right)}{\sqrt{N_{w}}}\right\} \\
& =Q\left[\frac{\sqrt{2} A\left(h_{I}+h_{Q}\right)}{\sqrt{N_{w}}}\right] \\
P_{2} & =P_{r}\left\{A\left(h_{I}-h_{Q}\right)+w_{I}<0\right\} P_{r}\left\{A\left(h_{I}+h_{Q}\right)+w_{Q}<0\right\} \\
& =Q\left[\frac{\sqrt{2} A\left(h_{I}-h_{Q}\right)}{\sqrt{N_{w}}}\right] Q\left[\frac{\sqrt{2} A\left(h_{I}+h_{Q}\right)}{\sqrt{N_{w}}}\right]
\end{aligned}
$$

The conditional bit error probabilities $P_{a}, P_{b}, P_{c}$ are computed as

$$
\begin{aligned}
P_{a} & =\frac{1}{4}\left[n_{a 1 I} \cdot P_{1 I}+n_{a 1 Q} \cdot P_{1 Q}+n_{a 2} \cdot 2 \cdot P_{2}\right] \\
& =\frac{1}{4}\left[2 P_{1 I}+2 P_{1 Q}+8 P_{2}\right] \\
P_{b} & =\frac{1}{4}\left[n_{b 1 I} \cdot P_{1 I}+n_{b 1 Q} \cdot P_{1 Q}+n_{b 2} \cdot 2 \cdot P_{2}\right] \\
& =\frac{1}{4}\left[P_{1 I}+2 P_{1 Q}+4 P_{2}\right] \\
P_{c} & =\frac{1}{4}\left[n_{c 1 I} \cdot P_{1 I}+n_{c 1 Q} \cdot P_{1 Q}+n_{c 2} \cdot 2 \cdot P_{2}\right] \\
& =\frac{1}{4}\left[P_{1 I}+P_{1 Q}+2 P_{2}\right]
\end{aligned}
$$

where $n_{a 1 I}, n_{a 1 Q}$ denotes the number of non-diagonal neighbouring regions that differ in 1 bit along the I/Q axis from the transmitted type $a$ symbol, and $n_{a 2}$ is the number of diagonal neighbouring regions that differ in 2 bits from the transmitted type $a$ symbol. $n_{b 1 I}, n_{b 1 Q}, n_{b 2}, n_{c 1 I}, n_{c 1 Q}, n_{c 2}$ are defined similarly for type $b$ and type $c$ symbols. The bit error probability is thus

$$
\begin{aligned}
P_{b}= & \frac{1}{16}\left(n_{a} P_{a}+n_{b} P_{b}+n_{c} P_{c}\right)=\frac{1}{16}\left(4 P_{a}+8 P_{b}+4 P_{c}\right) \\
= & \frac{1}{16}\left(5 P_{1 I}+7 P_{1 Q}+18 P_{2}\right) \\
= & \frac{5}{16} Q\left[\frac{\sqrt{2} A\left(h_{I}-h_{Q}\right)}{\sqrt{N_{w}}}\right]+\frac{7}{16} Q\left[\frac{\sqrt{2} A\left(h_{I}+h_{Q}\right)}{\sqrt{N_{w}}}\right] \\
& +\frac{18}{16} Q\left[\frac{\sqrt{2} A\left(h_{I}-h_{Q}\right)}{\sqrt{N_{w}}}\right] Q\left[\frac{\sqrt{2} A\left(h_{I}+h_{Q}\right)}{\sqrt{N_{w}}}\right]
\end{aligned}
$$

Denote $x=h_{I}-h_{Q}, y=h_{I}+h_{Q}$. The PDFs of $x, y$ are given in (11). The bit error probability can be expressed as

$$
\begin{aligned}
P_{b}(x, y)= & \frac{5}{16} Q\left[\frac{\sqrt{2} A x}{\sqrt{N_{w}}}\right]+\frac{7}{16} Q\left[\frac{\sqrt{2} A y}{\sqrt{N_{w}}}\right] \\
& +\frac{18}{16} Q\left[\frac{\sqrt{2} A x}{\sqrt{N_{w}}}\right] Q\left[\frac{\sqrt{2} A y}{\sqrt{N_{w}}}\right]
\end{aligned}
$$

where $N_{w}=P_{1}+P_{2}+N_{0}$. The average bit error probability $\bar{P}_{b}$ is derived by taking expectation of $P_{b}(x, y)$ expressed in (16) with respect to $x$ and $y$. Going through the same procedure as we did earlier for QPSK modulation, we have

$$
\begin{aligned}
& P_{b}(y)=\frac{5}{32}+Q\left[\frac{\sqrt{2} A y}{\sqrt{N_{w}}}\right] \\
& \bar{P}_{b}=\mathrm{E}\left[P_{b}(y)\right]=\frac{5}{32}+Q\left(\frac{\mu}{\sqrt{1+\lambda^{2}}}\right)
\end{aligned}
$$

where $\lambda=\frac{\sqrt{2} A \sigma}{\sqrt{N_{w}}}, \mu=\frac{\sqrt{2} A m_{y}}{\sqrt{N_{w}}}$.

\section{Analytical Results and Performance COMPARISON}

Comparison between analytical and simulated results is presented in this section to verify theoretical analysis conducted in the previous sections. The simulated curves are obtained numerically by averaging the results over 1000 channel realizations. Some statistics of the SUI-3 channel coefficients are summarized below:

$$
\begin{aligned}
& \mathrm{E}\left[h_{0}\right]=\sqrt{0.5}+j \sqrt{0.5} \\
& P_{0}=\mathrm{E}\left[\left|h_{0}\right|^{2}\right]=1.5 \\
& \operatorname{var}\left[h_{0}\right]=\mathrm{E}\left[\left|h_{0}\right|^{2}\right]-\left|\mathrm{E}\left[h_{0}\right]\right|^{2}=0.5 \\
& \mathrm{E}\left[h_{1}\right]=\mathrm{E}\left[h_{2}\right]=0 \\
& P_{1}=\operatorname{var}\left[h_{1}\right]=\mathrm{E}\left[\left|h_{1}\right|^{2}\right]=10^{-0.5}=0.3162 \\
& P_{2}=\operatorname{var}\left[h_{2}\right]=\mathrm{E}\left[\left|h_{2}\right|^{2}\right]=0.1
\end{aligned}
$$

Based on the above information, $\sigma^{2}=0.25, s^{2}=m_{1}^{2}+m_{2}^{2}=$ $0.5+0.5=1$ in equation (2), (4), (7), (8) and $\sigma^{2}=0.25 * 2=$ $0.5, m_{y}=\sqrt{0.5}+\sqrt{0.5}=2 \sqrt{0.5}$ in equation (11), (15), (17).

Fig. 3 shows that the theoretical analysis for coherent QPSK modulation expressed by equation (4) is in close agreement with the simulated results, even with imperfect phase estimate which is produced by maximum likelihood algorithm presented in [9]. The figure also shows the close agreement between theoretical analysis for non-coherent QPSK expressed by (15) and the simulated results. It is evident that the coherent detection greatly improves the system performance compared to the non-coherent scheme. However, coherent algorithm alone can not remove the effect the intersymbol interference (ISI) which is inherent in multipath BFWA channel.

For 16-QAM illustrated in Fig. 2, the average symbol energy is

$$
E_{s}=\frac{1}{4}\left[\left(A^{2}+A^{2}\right)+2\left(A^{2}+9 A^{2}\right)+\left(9 A^{2}+9 A^{2}\right)\right]=10 A^{2}
$$




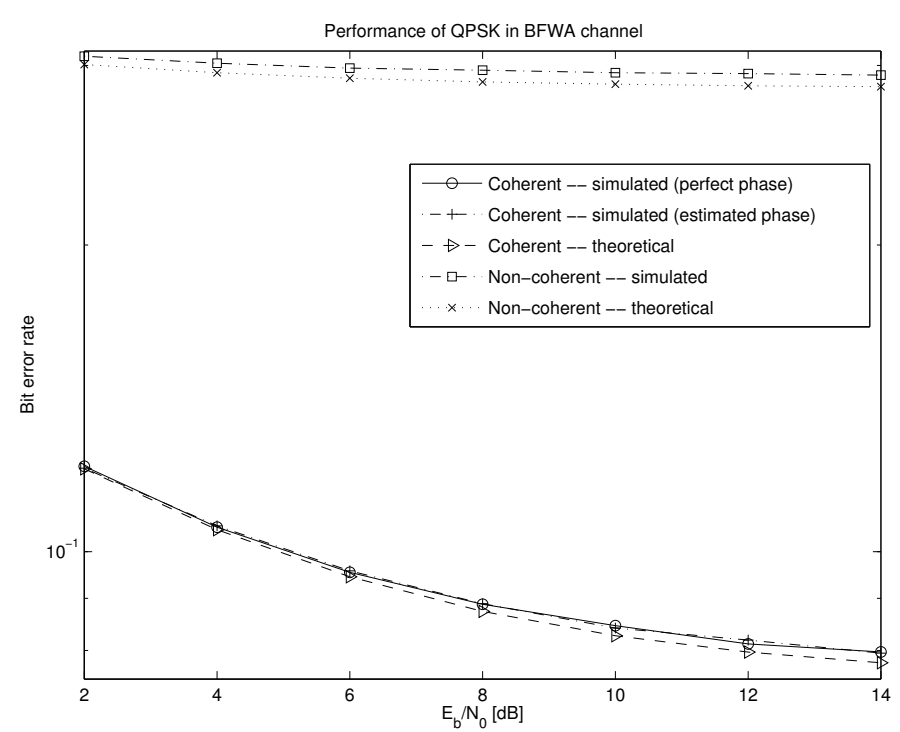

Fig. 3. Coherent vs. non-coherent detection for QPSK modulated BFWA system.

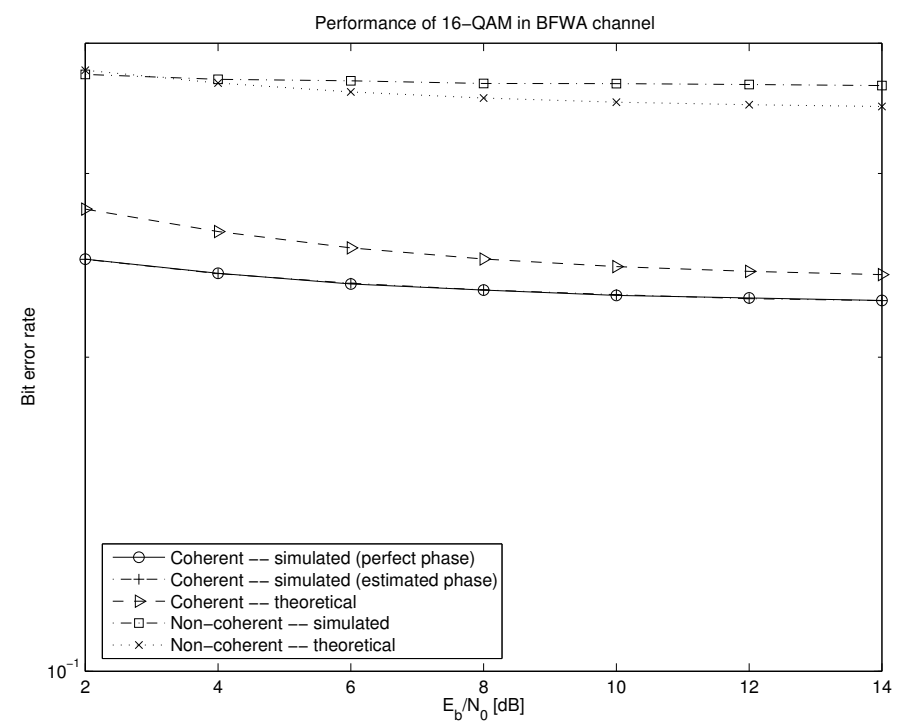

Fig. 4. Coherent vs. non-coherent detection for 16-QAM modulated BFWA system.

In our simulations, the transmitted symbols are normalized to unit average energy, therefore, $E_{s}=10 A^{2}=1, A=\sqrt{0.1}$ in equation (7) and in (17). Fig. 4 shows slight discrepancy between the theoretical analysis for coherent 16-QAM modulation expressed by (7), (8) and the simulated results. The gap is quite small though. Coherent detection with imperfect phase estimate yields very close performance compared to the genie-aided case assuming perfect knowledge of the channel. Fig. 4 also shows the close agreement between theoretical analysis for non-coherent 16-QAM expressed by (17) and the simulated results.

Comparing Fig. 3 with Fig. 4, it is evident that QPSK modulation is more robust but supports lower data rate, while 16-QAM supports higher data rate but has worse performance. Their performance gap is much more obvious with coherent detection than with non-coherent detection.

\section{Conclusions}

The QPSK and 16-QAM modulated BFWA systems with conventional detection are theoretically analyzed in this paper. Comparison with the simulated results show that the analysis is fairly accurate. Both simulation and analysis indicate that the BFWA channels are very hostile. Conventional non-coherent and coherent detection schemes will therefore not suffice. Channel coding and equalization algorithms are needed to remove the detrimental effect of ISI and improve the system performance. They are the future research topics for the authors.

\section{REFERENCES}

[1] H. Bolcskei. "Fixed Broadband Wireless Access: State of the Art, Challenges, and Future Directions". IEEE Communication Magazine, pp. 100-108, Jan. 2001.

[2] IEEE 802.16 Working Group on Broadband Wireless Access Standards. available at http://grouper.ieee.org/groups/802/16/. 2002.

[3] Draft ETSI TS 101, Broadband Radio Access Networks (BRAN), HIPERMAN. available at http://portal.etsi.org/bran/Summary.asp, 2002.

[4] V. Erceg. "An empirically based path loss model for wireless channels in suburban environments". IEEE JSAC, vol. 17, no. 7, pp. 1205-1211, July 1999.

[5] J. Proakis. Digital Communications, 3rd edition, McGraw-Hill, 1995.

[6] J. Holtzman. "A simple, accurate method to calculate spreadspectrum multiple-access error probabilities". IEEE Transactions on Communications, vol. 40, no. 3, pp. 461-464, March 1992.

[7] Alan Jefferey. Handbook of Mathematical Formulas and Integrals, Academic Press, 2nd edition.

[8] S. Verdu. Multiuser Detection, 1st edition, Cambridge University Press, 1998

[9] P. Xiao, R. Carrasco, I. Wassell. "Linear algebraic approach to turbo equalization". Submitted to IEEE Transactions on Communications. 